\title{
Nocturnal heart rate variability in 1-year-old 1 crasenat infants analyzed by using the Least Square Cosine Spectrum Method
}

\author{
Yuko Kochiya*, Akari Hirabayashi and Yuhei Ichimaru
}

\begin{abstract}
Background: To evaluate the dynamic nature of nocturnal heart rate variability, RR intervals recorded with a wearable heart rate sensor were analyzed using the Least Square Cosine Spectrum Method.

Methods: Six 1-year-old infants participated in the study. A wearable heart rate sensor was placed on their chest to measure RR intervals and 3-axis acceleration. Heartbeat time series were analyzed for every $30 \mathrm{~s}$ using the Least Square Cosine Spectrum Method, and an original parameter to quantify the regularity of respiratory-related heart rate rhythm was extracted and referred to as "RA (RA-COSPEC: Respiratory Area obtained by COSPEC)." The RA value is higher when a cosine curve is fitted to the original data series.

Results: The time sequential changes of RA showed cyclic changes with significant rhythm during the night. The mean cycle length of RA was $70 \pm 15$ min, which is shorter than young adult's cycle in our previous study. At the threshold level of RA greater than 3, the HR was significantly decreased compared with the RA value less than 3.

Conclusions: The regularity of heart rate rhythm showed dynamic changes during the night in 1-year-old infants. Significant decrease of HR at the time of higher RA suggests the increase of parasympathetic activity. We suspect that the higher RA reflects the regular respiratory pattern during the night. This analysis system may be useful for quantitative assessment of regularity and dynamic changes of nocturnal heart rate variability in infants.
\end{abstract}

Keywords: Infant, Heart rate variability, Rhythm analysis, Nighttime, Least Square Cosine Spectrum Method

\section{Background}

Polysomnography (PSG) is the objective standard method to evaluate the sleep pattern with high accuracy; it measures brain wave activity, eye movements, muscle activity, heart rate, and respiration during sleep. However, this procedure is time-consuming, requires instruments to monitor several physiological signals, needs specific skills, and is usually performed by specially trained technicians in restricted environments such as sleep laboratories. Therefore, its use in evaluating infants and young children is relatively limited. Several sleep studies have used subjective assessment tools for the evaluation of sleep conditions in children. These were performed using a questionnaire under free-living conditions. However, this method remains limited because child behavior must be observed continuously by the

\footnotetext{
* Correspondence: kochiya@tokyo-kasei.ac.jp

Tokyo Kasei University, 1-18-1 Kaga, Itabashi-ku, Tokyo 173-8602, Japan
}

parent. Recently, a small, light-weight, wearable device has been developed to simultaneously record heart rate, 3 -axis acceleration, and body temperature. This device can conveniently measure the RR interval, body position, and body movements under free-living conditions over $24 \mathrm{~h}$. The beat-to-beat heart rate variability (HRV), determined from the RR intervals, is widely used as a noninvasive tool for assessing autonomic nervous function.

Autonomic nervous function changes are modified by sleep stages [1-4]. Thus, autonomic nervous function during sleep has recently been used to identify sleep stages in adults. However, the autonomic nervous system is immature in infants. They have a fast heart rate and rapid shallow breathing compared with adults. Although several studies have investigated the developmental changes of autonomic nervous function using HRV [5, 6], few studies have evaluated the dynamics of nocturnal changes of autonomic nervous function in infants. 
With spectral analysis of HRV, the power spectrum contains two main frequency components: a low-frequency component (LF, $0.04-0.15 \mathrm{~Hz}$ ) and a high-frequency component (HF, $0.15-0.50 \mathrm{~Hz}$ ). The HF component is equivalent to respiratory sinus arrhythmia (RSA) and is mediated solely by parasympathetic activity [7]. RSA is one of the sinusoidal rhythms of the heartbeat in our pathophysiological system. It represents beat-to-beat fluctuations in heart rate associated with the respiratory cycle; the heart rate accelerates during inspiration and decelerates during expiration [8]. Fast Fourier transformation (FFT) is a powerful method to extract the spectral components of HRV and has been used in many studies that have investigated autonomic nervous system activity during sleep. This analysis method, however, requires interpolation to produce an evenly sampled time series, and the original data number is limited to be an integer power of 2 . Then, analyses of HRV were usually performed at normal RR intervals of consecutive 5- or 10-min segments during the awake state, during stable NREM, and during REM sleep stages. In contrast, the Least Square Cosine Spectrum Method is useful to directly and quantitatively analyze the biological rhythm $[9,10]$. This method has the advantage of being able to estimate the cosine components of data composed of unequal intervals and to arbitrarily configure the window range of analysis. This analysis method was applied with a window range of $30 \mathrm{~s}$ of heartbeat time series, which corresponded to PSG analysis epochs. In our previous study, we developed an original parameter to quantify the regularity of heart rate rhythm which reflects the respiratory pattern and referred to as "RA" [11]. We applied the Least Square Cosine Spectrum Method with a window range of $30 \mathrm{~s}$ of instantaneous heart rate in adults. The RA showed a 90-min rhythm during sleep, and the sensitivity and specificity for prediction of sleep stages 3 and 4 compared to PSG were 72.9 and $73.2 \%$, respectively [12]. The purpose of the present study was to investigate the nocturnal heart rate variability in infants using the algorithm we developed. The previous 24 -h period HR recording system was heavy, and it could therefore disturb infants' sleep. In this study, heart rate and 3axis acceleration were measured simultaneously using a wearable device.

\section{Methods}

\section{Subjects}

Six healthy infants (three females and three males), aged 12 months (range 11-13 months), participated in this study. All parents of the subjects were carefully instructed about the study, and all gave their written informed consent. The study was approved by the Tokyo-Kasei University Institutional Review Board.
Heart rate and 3-axis acceleration were continuously and simultaneously monitored using the wearable heart rate sensor "myBeat" (UNION TOOL CO., Tokyo, Japan). The device was placed on each subject's left chest with disposable electrode (Vitrode. T-50, Nihon Kohden Co., Tokyo, Japan). Measurement was conducted at subjects' homes under free-living conditions and started before bedtime and finished when the infants woke up in the morning. The parents were instructed to write down bedtime and waking times.

\section{Spectral analysis of HRV}

The RR intervals and acceleration signals were recorded in the device memory and transferred to a computer for analysis. The instantaneous heart rate was calculated from the RR interval sequences. False-negative or falsepositive RR intervals were excluded from the analysis. An automated computer analysis system was developed using Microsoft Visual Basic for Applications (VBA), and the Least Square Cosine Spectrum Method was applied with a window range of $30 \mathrm{~s}$ of the heartbeat time series. The formula of the cosine curve is as follows:

$$
Y=M+A \cos (2 \pi t / \omega-\theta)
$$

where $Y$ is the estimated cosine curve, $M$ is the MESOR (Midline Estimating Statistic Of Rhythm), $A$ is amplitude (a measure of half the extent of predictable variation within a cycle), $t$ is time, $\omega$ is period (duration of one cycle), and $\theta$ is acrophase (a measure of the time of overall high values recurring in each cycle).

By changing the period every $0.1 \mathrm{~s}$ sequentially, a high-frequency component of HRV was extracted. The cosine curve that showed the minimum value of the residual sum of squares calculated by subtracting the estimated cosine curve from the original data was referred to as the best-fit cosine curve. The series of the probability in each given cycle was calculated using the direct method by Sasaki $[13,14]$. Then, the reciprocal logarithm of the probability was calculated and defined as the RA (RA-COSPEC: Respiratory Area obtained by COSPEC), which is expressed as:

$$
\mathrm{RA}=\log (1 / \text { Probability })
$$

The value of RA is higher when a cosine curve is fitted to the original data series.

In addition, we analyzed fitting curve for the time sequential changes of RA by using the Least Square Cosine Spectrum Method when the period was changing from 30 to $120 \mathrm{~min}$. We calculated the probability of the fitness of cosine curve directly $[13,14]$. 


\section{Analysis of triaxial acceleration}

To determine the overall magnitude of physical activity, the vector magnitude $(G)$ was calculated by taking the square root of the sum of squares from each axis:

$$
G=\sqrt{\left(X^{2}+Y^{2}+Z^{2}\right)}
$$

where $G=9.8 \mathrm{~m} / \mathrm{s}^{2}$, the $X$-axis of triaxial acceleration reads right or left, the $Y$-axis reads up or down, and the $Z$-axis reads back or front movement. The sleeping position was estimated as in our previous study [15]: when the $Y$-axis value was more than -0.7 , the subject was considered to be in the lying position. In addition, the left lateral position was defined as an $X$-axis value $\geq 0.5 \mathrm{G}$, the right lateral position was defined as an $X$-axis value $<-0.5 \mathrm{G}$, the supine position was defined as a $Z$-axis value $\geq 0.7 \mathrm{G}$, and the prone position was defined as a $Z$-axis value $<-0.7 \mathrm{G}$.

\section{Statistical analysis}

The $t$ test was used to examine the differences. $P$ value $<0.05$ was considered statistically significant.

\section{Results}

\section{Heart rate and cosine curve for $30 \mathrm{~s}$}

Figures 1 and 2 show an original data series of the instantaneous heart rate and the results of the data calculated from the cosine fitting curve for $30 \mathrm{~s}$. In Fig. 1, the period was $2.5 \mathrm{~s}$, the MESOR was $115 \mathrm{bpm}$, and the amplitude was $1.4 \mathrm{bpm}$. In contrast, the frequency and amplitude of the heart beat changes are more regular in Fig. 2, which shows the period, MESOR, and amplitude of $2.2 \mathrm{~s}, 106 \mathrm{bpm}$, and $8.4 \mathrm{bpm}$, respectively. The RA, indicating fitness for the cosine curve, was higher in Fig. 2 than in Fig. 1. In the present study, these parameters were calculated for nocturnal data at 30-s intervals.

\section{Dynamic changes of heart rate variability and acceleration}

Figure 3 illustrates the time sequential changes of period, amplitude, RA, mean value of instantaneous heart rate, 3-axis acceleration, and the vector magnitude

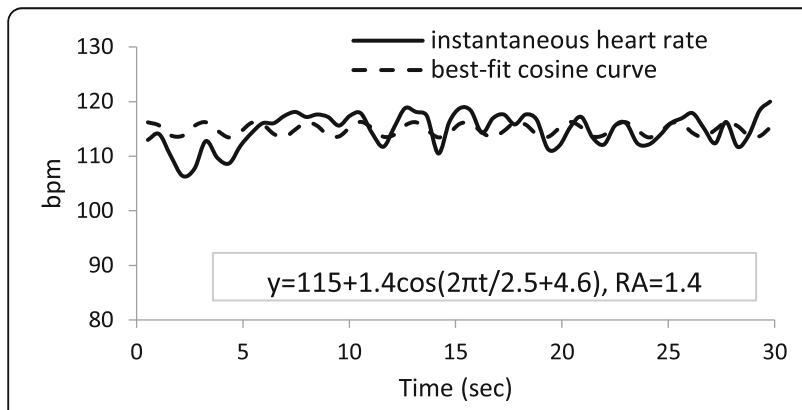

Fig. 1 Instantaneous heart rate and best-fit cosine curve at 21:54 PM

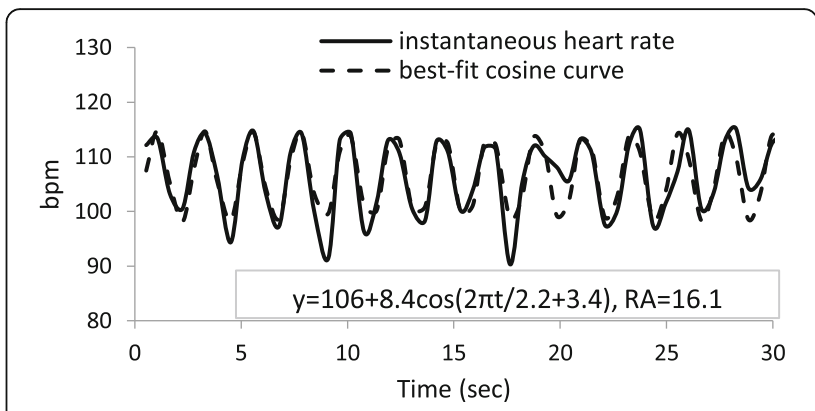

Fig. 2 Instantaneous heart rate and best-fit cosine curve at 20:08 PM

calculated every $30 \mathrm{~s}$ in one representative subject. As shown by the values of the $X$ and $Z$-axes, this subject spent more time in the prone position. The proportion of the supine position to time in bed was $6 \%$, the prone position was $54 \%$, the right lateral position was $3 \%$, and the left lateral position was $36 \%$ in this subject. The vector magnitude increased mostly accompanied with position changes. The RA was characterized by 11 times repeated increases or decreases during the night (Fig. 3, 3rd row). The cyclic changes of RA were observed with a cycle length of $64 \mathrm{~min}$, as measured by the Least Square Cosine Spectrum Method when the period was changing from 30 to $120 \mathrm{~min}$. The mean HR was $103.0 \pm 9.6 \mathrm{bpm}$ (Fig. 3, in 4th row). The HR changed inversely with RA, that is, it showed a decrease at the time of higher RA, and it increased with lower RA. In this case, the mean HR was $98.8 \pm 7.9 \mathrm{bpm}$ when the RA value was greater than 3 , but was $106.2 \pm 9.6 \mathrm{bpm}$ when was less than 3 . This analysis was performed for all subjects, and the results are presented in Table 1 . The HR was significantly lower when the RA was greater than 3 for all subjects.

For all subjects, mean time in bed by parental report was $606 \pm 66 \mathrm{~min}$. Figure 4 shows individual proportions of body position to time in bed. Among the six infants, four infants spent more than $50 \%$ of the time in bed in the prone position. The nocturnal individual profiles of RA are presented in Table 2 . The mean RA was $2.8 \pm 0.8$. The cyclic changes of RA with significant rhythm were obtained from all subjects $(p<0.001)$, and the mean cycle length of RA was $70 \pm 15 \mathrm{~min}$.

\section{Assessment of RSA amplitude}

The Least Square Cosine Spectrum Method is able to obtain the absolute value of amplitude, which approximates the original data if the estimated error of the cosine curve with the predicted period is smaller. The amplitude is used as an index of cardiac vagal activity. In this study, the mean values of period and amplitude were calculated when the probability as a function of cycle for a 30-s epoch was less than 0.001 , that is, RA 

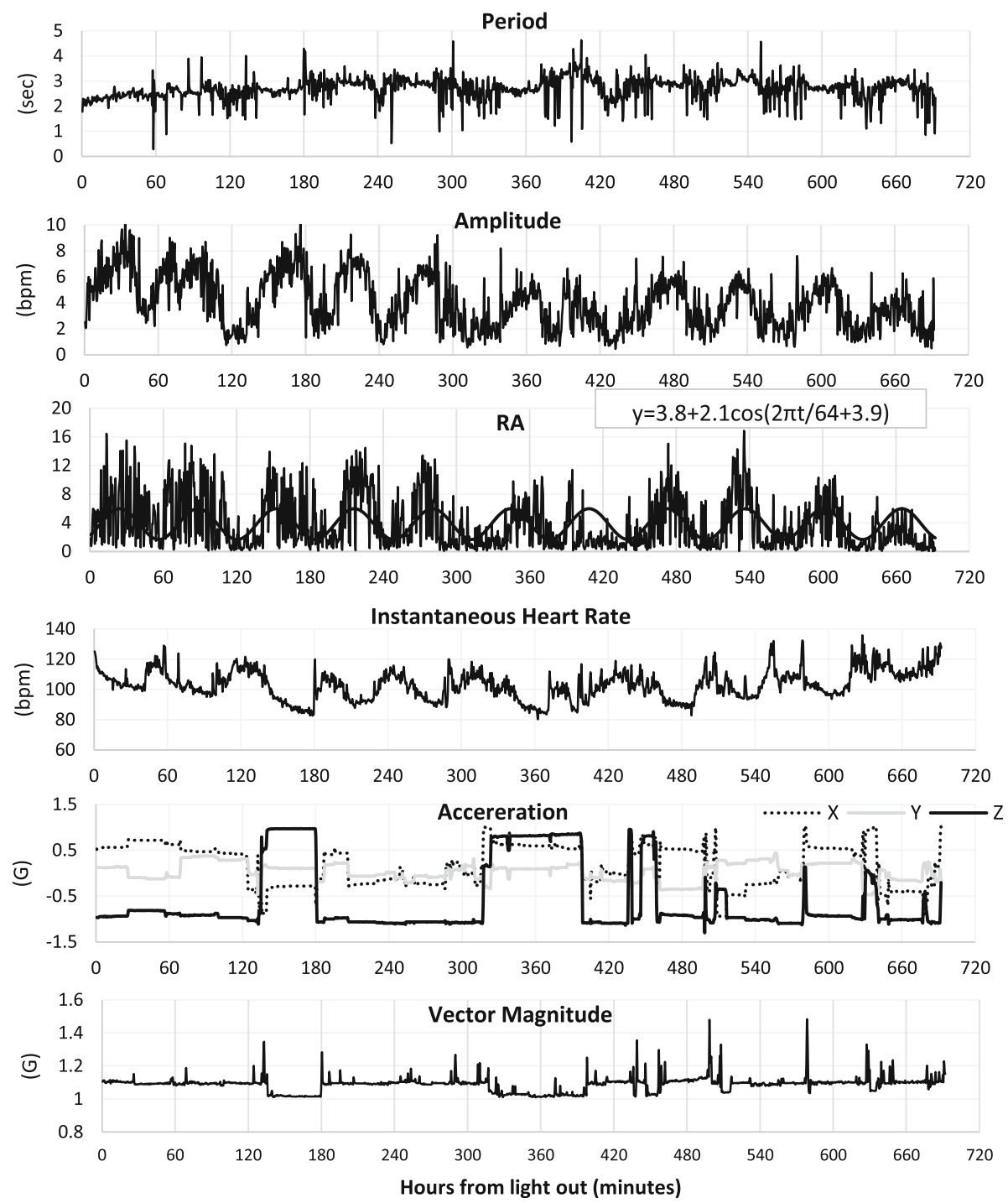

Fig. 3 Changes of period, amplitude, RA, HR, 3-axis acceleration, and vector magnitude for a representative subject

was greater than 3 . The results for all subjects are shown in Table 3. The period and amplitude values averaged $2.8 \pm 0.2 \mathrm{~s}$ and $4.2 \pm 1.1 \mathrm{bpm}$ for the six subjects, respectively. The amplitude differed among individuals; the range extended from 2.7 to $5.4 \mathrm{bpm}$.

\section{Discussion}

In this paper, we investigated the dynamic nature of nocturnal heart rate variability by using the Least Square Cosine Spectrum Method in 1-year-old infants and extracted a parameter referred to as "RA" to quantify the

Table 1 The comparison of mean HR when the RA was greater or less than the value 3

\begin{tabular}{llll}
\hline Subject & Mean HR when the RA value $\geq 3(\mathrm{bpm})$ & Mean HR when the RA value $<3(\mathrm{bpm})$ & $P$ value \\
\hline A & $88.9 \pm 5.6(n=273)$ & $92.3 \pm 9.0(n=1079)$ & $<0.001$ \\
B & $104.4 \pm 7.8(n=336)$ & $114.4 \pm 11.1(n=838)$ & $<0.001$ \\
C & $105.8 \pm 6.4(n=323)$ & $115.1 \pm 6.4(n=741)$ & $<0.001$ \\
D & $89.3 \pm 6.2(n=275)$ & $94.3 \pm 10.7(n=722)$ & $<0.001$ \\
E & $102.6 \pm 3.4(n=510)$ & $109.1 \pm 9.3(n=690)$ & $<0.001$ \\
F & $98.8 \pm 7.9(n=595)$ & $106.2 \pm 9.6(n=789)$ & $<.001$
\end{tabular}

We calculated the HR value when the RA was greater or less than 3 . Values are expressed as mean \pm SD. The difference of HR was assessed using the $t$ test, and a $P$ value $<0.05$ was considered statistically significant. There was statistically significant difference in all subjects $(P<0.001)$ 


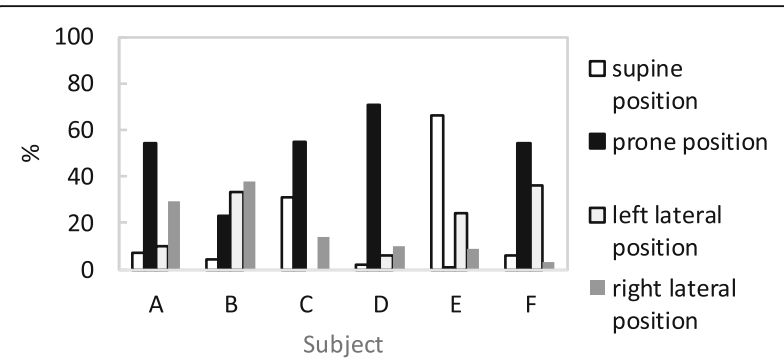

Fig. 4 Individual proportions of body position to time in bed for six infants

regularity of respiration-related heart rate rhythm. When heart rate time series fit well to cosine curve obtained from Least Square Cosine Spectrum Method, the RA value shown a higher value. This study demonstrated that the RA had repeated increases or decreases during the nighttime with the cycle length of $70 \mathrm{~min}$. When setting the threshold level of RA at 3, a significant decrease of $\mathrm{HR}$ was observed compared with the threshold value of below 3, suggesting the increase of parasympathetic activity at this period.

The respiratory pattern shows dynamic changes during sleep. Respiration becomes regular during NREM sleep and irregular during REM sleep [16]. Bond et al. [17], who investigated subjects from 16 to 69 years old, reported that there was a relatively precise correlation between the respiratory pattern and rhythmic HRV, with a very uniform pattern of frequency and amplitude of sinus arrhythmia in deep sleep (NREM sleep stages 3 and 4). On the other hand, in REM sleep, there was complete dissociation of respiration and rhythmic heart rate. In our previous study comparing the RA and sleep stage by using PSG recordings in young adults, the RA was lower during REM sleep, MT (Movement Time), and awake and was higher during sleep stages 3 and 4 [12]. Consequently, we suspect that the higher RA obtained from in the present study reflects the regular respiratory pattern especially observed during deep sleep.

Table 2 Individual nocturnal profiles of RA for six infants

\begin{tabular}{llll}
\hline Subject & Mean of RA & Max of RA & Cycle length of RA (min) \\
\hline A & 1.9 & 13.0 & 62 \\
B & 2.3 & 15.2 & 77 \\
C & 3.0 & 17.9 & 97 \\
D & 2.3 & 12.5 & 58 \\
E & 3.5 & 17.2 & 61 \\
F & 3.8 & 16.8 & 64 \\
Average & 2.8 & 15.4 & 70 \\
SD & 0.8 & 2.3 & 15 \\
\hline
\end{tabular}

The RA value during the night averaged $2.8 \pm 0.8$ for the six subjects. The cyclic changes of RA were obtained from all subjects, and the mean cycle length of RA was $70 \pm 15 \mathrm{~min}$
Table 3 The period and amplitude of the cosine curve when the RA value was greater than 3

\begin{tabular}{lll}
\hline Subject & Period (sec) & Amplitude (bpm) \\
\hline A $(n=273)$ & 2.8 & 5.0 \\
B $(n=336)$ & 2.8 & 3.5 \\
C $(n=323)$ & 3.2 & 3.8 \\
D $(n=275)$ & 2.6 & 5.0 \\
E $(n=510)$ & 2.7 & 2.7 \\
F $(n=595)$ & 2.7 & 5.4 \\
Average & 2.8 & 4.2 \\
SD & 0.2 & 1.1
\end{tabular}

To evaluate the RSA amplitude used as an index of cardiac vagal activity, we calculated when the RA was greater than 3 . The period and amplitude values averaged $2.8 \pm 0.2 \mathrm{~s}$ and $4.2 \pm 1.1 \mathrm{bpm}$ for the six subjects, respectively. The amplitude differed among individuals; the range extended from 2.7 to $5.4 \mathrm{bpm}$

Concerning the period of cyclic changes of RA, the mean value in young adults was previously found to be $89 \pm 11 \mathrm{~min}$ [11], while it was $70 \pm 15 \mathrm{~min}$ in 1-year-old infants in the present study. Sleep alternates between REM and NREM sleep (akin to active and quiet sleep in infants). In young adults, REM sleep comprises about $20 \%$ of total sleep time, but in newborn infants, it comprises about $50 \%$ and decreases with age. The duration of the REM-NREM sleep cycle is generally thought to be about 90 min in young adults, but an infant's cycle is shorter, lasting 40 to $60 \mathrm{~min}$ in newborn babies and infants, 60 to $80 \mathrm{~min}$ in 2- to 5 -year-olds, and close to adult levels at 5 to 10 years [18]. A future study is required to clarify the possibility of the RA for estimating sleep cycle in infants.

Furthermore, another advantage of the Least Square Cosine Spectrum Method is that it is able to obtain the absolute value of the amplitude, which is used as an index of cardiac vagal activity. In this study, the mean amplitude of the cosine fitting curve was $4.2 \pm 1.1 \mathrm{bpm}$ when the RA was greater than 3. We should, in future, examine the useful method for evaluating the agerelated changes of absolute amplitude in consideration of the differentiation of mean heart rate and respiratory rate between infants and adults. A limitation of this study is the small sample size, which was restricted to 1year-old infants. Further studies including a larger sample are necessary to investigate developmental changes in autonomic nervous function. However, one should take into account that RSA amplitude is not only affected by vagal tone but also by respiratory frequency and tidal volume $[19,20]$.

Chronobiological studies have demonstrated that cardiovascular events occur frequently during night time or sleep time. Autonomic nervous function changes have been implicated in the development of cardiovascular disease. In addition, the association between infant sleeping 
position and risk of sudden infant death syndrome (SIDS) has been investigated. The method used in the present study could be used for the assessment of not only heart rate variability but also body position and body movement. Among the six infants, four infants spent more than $50 \%$ of the time in bed in the prone position. Hence, the application of the method to epidemiological studies investigating relationships between sleep position and autonomic nervous system function is expected.

\section{Conclusions}

This study investigated the nocturnal heart rate variability in 1-year-old infants using the Least Square Cosine Spectrum Method. We extracted a parameter to quantify the regularity of respiration-related heart rate rhythm and referred to as "RA." Our study indicates that (1) the RA showed cyclic changes with significant rhythm during the night, (2) the mean cycle length of RA was $70 \mathrm{~min}$, and (3) the HR decreased at the time of higher RA, suggesting the increase of parasympathetic activity. We suspect that the higher RA reflects the regular respiratory pattern during the night. This analysis system may be useful for quantitative assessment of regularity and dynamic changes of nocturnal heart rate variability in infants.

\section{Abbreviations}

FFT: Fast Fourier transformation; HF: High frequency; HRV: Heart rate variability; LF: Low frequency; PSG: Polysomnography; RSA: Respiratory sinus arrhythmia; SIDS: Sudden infant death syndrome; VBA: Visual Basic for Applications

\section{Acknowledgements}

Not applicable

\section{Funding}

This work was supported by the graduate school of Tokyo-Kasei University for the Research Development Project B.

\section{Availability of data and materials}

The individual raw data will not be shared publicly because of the ethics policy.

\section{Authors' contributions}

$\mathrm{YK}, \mathrm{AH}$, and $\mathrm{Yl}$ contributed to the study design, data acquisition, analysis, and interpretation of data. YK drafted the manuscript. AH and YI checked the manuscript. All authors have approved the final manuscript.

\section{Ethics approval and consent to participate}

All parents of the subjects were carefully instructed about the study, and all gave their written informed consent. The study was approved by the TokyoKasei University Institutional Review Board.

\section{Consent for publication}

All parents of the subjects gave written informed consent including publication of results.

\section{Competing interests}

The authors declare that they have no competing interests.

\section{Publisher's Note}

Springer Nature remains neutral with regard to jurisdictional claims in published maps and institutional affiliations.
Received: 23 August 2016 Accepted: 8 September 2017

Published online: 16 September 2017

\section{References}

1. Ichimaru Y, Clark KP, Ringler J, Weiss WJ. Effect of sleep stage on the relationship between respiration and heart rate variability. Computers in Cardiology 1990, proceedings: IEEE; 1990. p. 657-60.

2. Bonnet MH, Arand DL. Heart rate variability: sleep stage, time of night, and arousal influences. Electroencephalogr Clin Neurophysiol. 1997;102:390-6.

3. Ako M, Kawara T, Uchida S, Miyazaki S, Nishihara K, Mukai J, Hirao K, Ako J, Okubo Y. Correlation between electroencephalography and heart rate variability during sleep. Psychiatry Clin Neurosci. 2003;57:59-65.

4. Busek P, Vankova J, Opavsky J, Salinger J, Nevsimalova S. Spectral analysis of heart rate variability in sleep. Physiol Res. 2005;54:369-76.

5. Finley JP, Nugent ST. Heart rate variability in infants, children and young adults. J Auton Nerv Syst. 1995;51:103-8.

6. Korkushko OV, Shatilo VB, Plachinda YI, Shatilo TV. Autonomic control of cardiac chronotropic function in man as a function of age: assessment by power spectral analysis of heart rate variability. J Auton Nerv Syst. 1991;32:191-8.

7. Pomeranz B, Macaulay RJB, Caudill MA, Kutz I, Adam D, Gordon D, Kilborn KM, Barger AC, Shannon DC, Cohen RJ, Benson H. Assessment of autonomic function in humans by heart rate spectral analysis. Am J Phys. 1985;248:H151-3.

8. Ludwig C. Beitrage zur Kenntniss des Einflusses Respirationsbewegungen auf den Blutlauf im Aortensysteime. Arch Anat Physiol. 1947;13:242-302.

9. Halberg F, Lagoguey M, Reinberg A. Human circannual rhythms over a broad spectrum of physiological processes. Int J Chronobiol. 1983:8:225-68.

10. Ichimaru Y. Least square multivariate cosine spectrum analysis for ambulatory blood pressure and heart rate. Ther Res. 1993;14:194-201.

11. Kochiya Y, Ichimaru Y. Development of circadian respiratory and heart rate rhythm analysis system by using cosine spectrum method. Jpn J Physiol Anthropol. 2009;14:15-20.

12. Kochiya $Y$, Ichimaru $Y$. Estimation of sleep stages by using heart rate rhythm analysis. Jpn J Physiol Anthropol. 2010;15:91-5.

13. Sasaki Y. Some considerations of the rhythmicity test. Excerpta Med ICS. 1973;300:105.

14. Sasaki T, Chiba Y, editors. Chronobiology. Tokyo: Asakura publishing; 1979. p. $325-8$.

15. Hirabayashi A, Kochiya Y, Ichimaru Y. Examination and application of estimation method for body postures in daily life using triaxial accelerometers. Jpn J Physiol Anthropol. 2015;20:187-96.

16. Martin RJ. Cardiorespiratory disorders during sleep. New York: Futura Publishing Company; 1984. p. 21.

17. Bond WC, Bohs C, Ebey J, Wolf S. Rhythmic heart rate variability (Sinus arrhythmia) related to stages of sleep. Cond Reflex. 1973;8:98-107.

18. The Japanese Society of Sleep Research. Handbook of Sleep Science and Sleep Medicine. Tokyo: Asakura publishing; 1994. p. 24

19. Angelone A, Coulter NA. Respiratory sinus arrhythmia: a frequency dependent phenomenon. J Appl Physiol. 1964;19:479-82.

20. Hirsch JA, Bishop B. Respiratory sinus arrhythmia in humans: how breathing pattern modulates heart rate. Am J Phys. 1981;241:H620-9.

Submit your next manuscript to BioMed Central and we will help you at every step:

- We accept pre-submission inquiries

- Our selector tool helps you to find the most relevant journal

- We provide round the clock customer support

- Convenient online submission

- Thorough peer review

- Inclusion in PubMed and all major indexing services

- Maximum visibility for your research 